\title{
A Progressive Image Transmission Method Based on Discrete Wavelet Transform (DWT)
}

\author{
Md. Rifat Ahmmad Rashid \\ CSE Discipline, Khulna University, Khulna, Bangladesh \\ rifat.abir@gmail.com \\ Mir Tafseer Nayeem \\ Department of Computer Science and Information Technology (CIT) \\ Islamic University of Technology (IUT) \\ Board Bazar, Gazipur-1704, Bangladesh \\ mtnayeem@yahoo.com \\ Kamrul Hasan Talukder \\ Dept. of Information Engineering, Hiroshima University, Japan. \\ kht@ hiroshima-u.ac.jp \\ Md. Saddam Hossain Mukta \\ Department of Computer Science and Information Technology (CIT) \\ Islamic University of Technology (IUT) \\ Board Bazar, Gazipur-1704, Bangladesh \\ mukta944@gmail.com
}

\begin{abstract}
In this paper, a wavelet-based progressive image transmission (PIT) scheme is proposed. Here a combined method is proposed to reduce the image browsing time. The proposed scheme transforms a digital image from spatial domain into frequency domain by using discrete wavelet transformation. For wavelet transformation phase we have used Haar wavelet transformation. But it is computationally rigorous. Using concurrent computing we have significantly reduced computation time overhead as well as transmission time. According to the experimental results, the proposed scheme provides the accuracy of reconstructed image and the image browsing time reduces significantly.
\end{abstract}

Index Terms - Discrete wavelet Transform (DWT), Concurrent Computing, PIT System

\section{INTRODUCTION}

The regular scanning pattern traditionally used in image transmission means that image pixels are displayed from left to right and top to bottom. Often the image is only recognizable when a substantial portion of data has been transmitted. Considering that significant amounts of data are needed to represent large digital images, image transmission across low-bandwidth channels can be exceedingly slow. One method to reduce transmission time is image compression, which can significantly reduce an image's file size using lossy coding. However, the compression ratio is limited by the image quality degradation that can be tolerated. High- quality large images sent over low-bandwidth links will still incur a lengthy transmission time (Ho et al., 1994). Furthermore, with a number of image compression schemes such as some segmentation-based and fractal based methods, the whole compressed data file must be received and decoded before any part of the image can be displayed [3]. This represents a sizeable delay before the image can be viewed. A better solution is to improve the transmission method as well so that the image is transmitted progressively. Thus using PIT, users can get a glance of the image earlier and can decide whether it is the correct image or abort it. In case of large size images, the encoding and decoding processes for transmitting image also takes mentionable time, because the traditional way followed for these are computationally rigorous. Using PIT, users can preview the image in advance and therefore decide whether to abort the transferring process or wait for the image to be refined. These advantages and the growing popularity of network multimedia systems have led to the development of a wide variety of PIT techniques. PIT methods can be divided into four major classes: successive approximation methods, transmission sequence-based methods, multistage residual quantization methods, and multi resolution or hierarchical coding methods [4, 10]. The target of PIT is to encode the original image into a data stream from which image can be reconstructed efficiently. Also it should provide a fast glance of the image to the user. The encoding and decoding processes should be manipulated such a way that it fulfills the criteria [2]. We have proposed a scheme where concurrent computing has used in both encoding and decoding phase to reduce the computation time $[3,8]$. 
Also concurrent technique is applied during the data stream transmission. Though concurrent computing technique has applied on both phases the quality of reconstructed image has been ensured. Here we have used Haar Discrete Wavelet Transformation to transform the digital image into the frequency domain to decompose the spatial pixels into frequency coefficients.

The results indicate that our proposed methodology provides the accuracy of reconstructed image and the image browsing time reduces significantly.

The remainder of the paper is organized as follows. In section II, a review of the Haar wavelet transform required to effectively implement our PIT system is discussed. The proposed progressive image transmission (PIT) system is described in Section III. After that, the proposed algorithm is discussed in section IV, and performance analysis is in section $\mathrm{V}$, finally we draw our conclusion in the last section.

\section{HaAR Wavelet Technique}

The discrete wavelet transform can be described as a series of filtering and sub sampling (decimating in time) as The Haar wavelet is the simplest type of wavelet. In discrete form, Haar wavelets are related to a mathematical operation called the Haar transform. the Haar wavelet is a certain sequence of functions. It is now recognized as the first known wavelet. This sequence was proposed in 1909 by Alfréd Haar. Haar used different functions to give an example of a countable orthonormal system for the space of squareintegrable functions on the real line [6]. As a special case of the Daubechies wavelet, it is also known as D2.The Haar wavelet is also the simplest possible wavelet. The technical disadvantage of the Haar wavelet is that it is not continuous, and therefore not differentiable [8].

The Haar wavelet's mother wavelet function $\psi(\mathbf{t})$ can be described as

$\psi(t)= \begin{cases}1 & 0 \leq t<\frac{1}{2} \\ -1 & \frac{1}{2} \leq t<1 \\ 0 & \text { otherwise }\end{cases}$

and its scaling function $\varphi(t)$ can be described as

$$
\varphi(t)=\left\{\begin{array}{cc}
1 & 0 \leq t<1 \\
0 & \text { otherwise }
\end{array}\right.
$$

Consider a 1D image with a resolution of four pixels, having values $\left[\begin{array}{llll}9 & 7 & 3 & 5\end{array}\right]$. Haar wavelet basis can be used to represent this image by computing a wavelet transform. To do this, first the average of the pixels together, pair wise, is calculated to get the new lower resolution image with pixel values [8 4]. Clearly, some information is lost in this averaging process. We need to store some detail coefficients to recover the original four pixel values from the two averaged values. Here 1 is chosen for the first detail coefficient, since the average computed is 1 less than 9 and 1 more than 7 . This single number is used to recover the first two pixels of the original four-pixel image.

TABLE I: DECOMPOSITION TO LOWER RESOLUTION

\begin{tabular}{|c|c|c|}
\hline Resolution & Averages & Detail Coefficients \\
\hline 4 & {$\left[\begin{array}{lll}9 & 7 & 3\end{array}\right]$} & \\
\hline 2 & {$\left[\begin{array}{lll}8 & 4\end{array}\right]$} & {$\left[\begin{array}{ll}1 & -1\end{array}\right]$} \\
\hline 1 & {$\left[\begin{array}{ll}6\end{array}\right]$} & {$[2]$} \\
\hline
\end{tabular}

Thus, for the one-dimensional Haar basis, the wavelet transform of the original four-pixel image is given by [ 6 21 -1]. We can reconstruct the image to any resolution by recursively adding and subtracting the detail coefficients from the lower resolution versions [5].

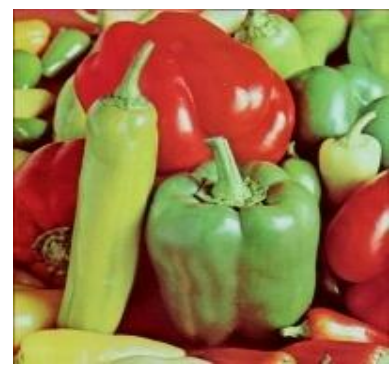

(a)

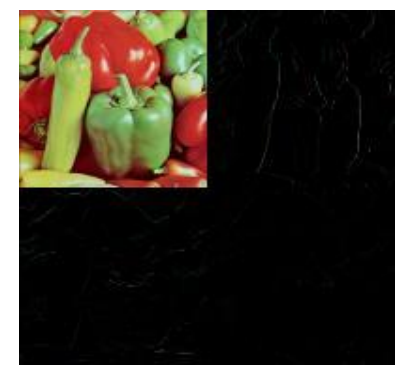

(b)
Figure 1. (a) Original image and (b) after 1 step of transformation

In summary, the main steps of the 2D image compression using wavelet as the basis functions are:

(a) Start with the matrix representing the original image.

(b) Compute the transformed matrix by the operation averaging and differencing (First for each row, then for each column).

(c) Choose threshold value $\varepsilon$ ( $\varepsilon=0$ for lossless and $\varepsilon=$ some + ve value for lossy).

(d) Replace all co-efficient of which is smaller than or equal to $\varepsilon$ by zero.

(e) Use the final matrix to compute the compression ratio and to reconstruct the original image as well.

\section{PROPOSED PIT SYSTEM}

Here is a description about how our progressive image transmission (PIT) system using concurrent computing technique works. There are several algorithms for wavelet based compression such as Embedded Zerotree Wavelet (EZW), Set Partitioning in Hierarchical Trees (SPHIT), Wavelet Difference Reduction (WDR), and Adaptively Scanned Wavelet Difference Reduction (ASWDR) etc. But here we have considered the very basic technique Haar Discrete Wavelet Transformation for color image transformation. 
a)

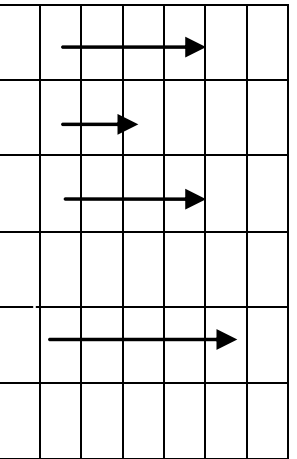

b)

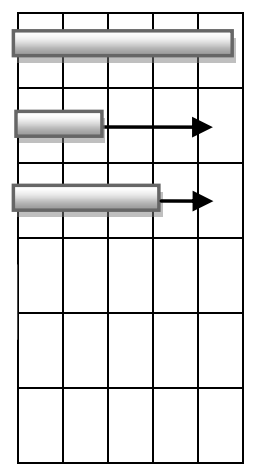

c)

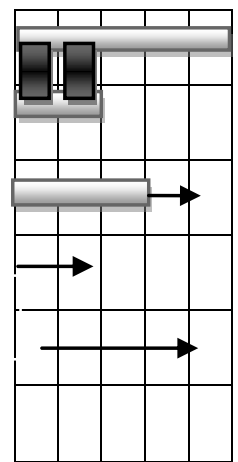

d)

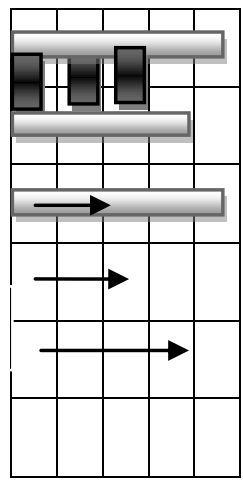

Figure 2. Concurrent transformation

a) Threads for each pair of row; b) Row transformation in progress; c) Column transformation done when pair of column elements is ready; d) Row and column transformation are in progress.

a)

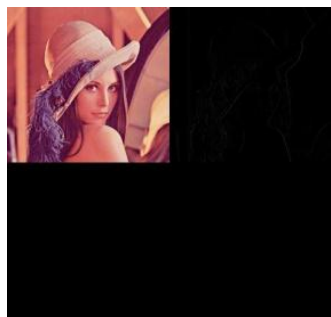

b)

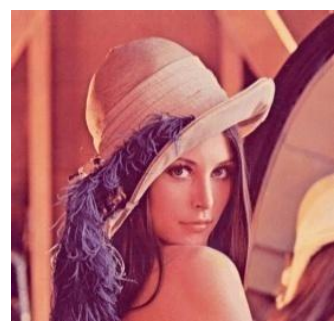

Figure 3. Image before transmitted (a) Original image (b) image result after encoding

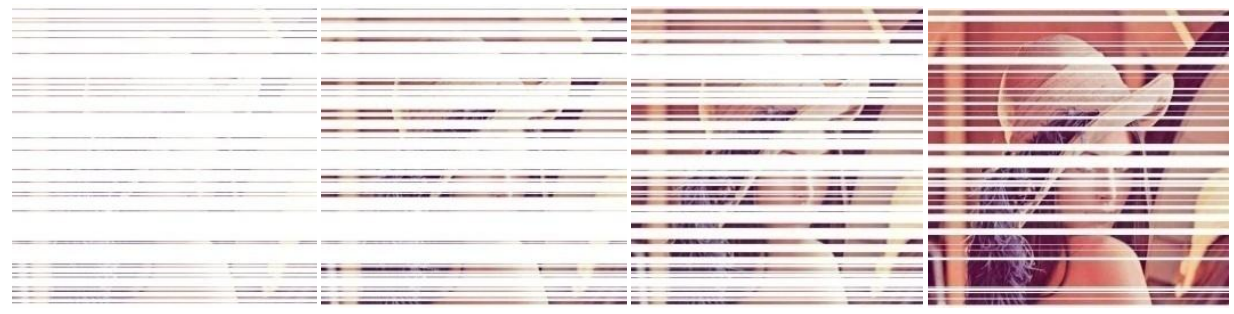

(iv) (i)

(iii)

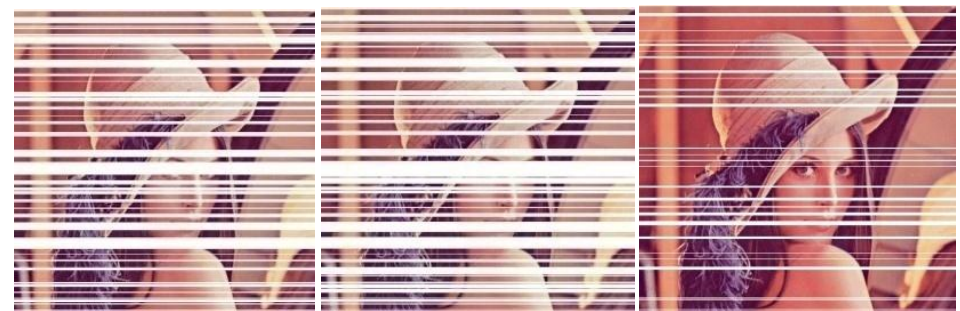

(v)

(vi)

(vii)

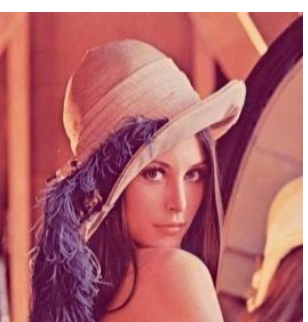

(viii)

Figure 4. Reconstructed image after receiving pairs of column concurrently, PSNR values

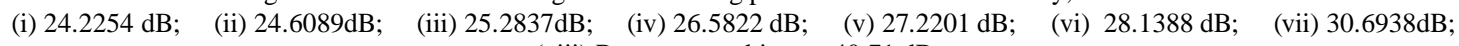

(viii) Reconstructed image $40.71 \mathrm{~dB}$

First of all the image matrix is mapped from the digital image. For row transformation, row transformation threads are initialized. We have used separate process for every pair of rows of the matrix.

The column transformation process is also embedded in the row transformation process. But we have used separate threads for transforming red, green and blue (RGB) components. Thus multiple threads are started at a time. The threads normally transform the rows following the discrete Haar wavelet transformation. But as soon as the pair of elements of the same column is transformed the column transformation for those two components is also done. This speeds up the transformation process as no waiting time required for the column transformation after row transformation has finished.

After completion of encoding process the matrix found with detail coefficients and average coefficients are found. Then the required thresh holding may be applied on it and the final matrix is ready to transmit to the receiver. At the transmission phase we have transmitted the matrix by pair of columns. This is also 
done by several threads. Thus while the receiver receives the data it receives columns randomly the decoding process is done as the reverse of encoding. Thus the reconstruction of image requires comparatively lower time.

For example we transmitted image lenna over $10 \mathrm{Kbps}$ line between two pc. Original image and encoded image given in figure 3 and steps of reconstructed image shown in figure 4.In figure 4 by PSNR value we showed the level of detail obtain after certain concurrent pairs of column available in the receiver and after Reconstruction of image.

\section{THE ALgORITHM}

A generalized algorithm for the proposed system is stated as below.

\section{Encoding Phase:}

- Initialize a thread for every pair of row of the image matrix. Use Separate thread for R, G and B component.

- When a pair of elements in the same column is available, transform them (column transform).

- If both row and column transformation for each bock ( 2 rows) is completed thread disposed.

- When all the available block (2 rows) transformation completed we get the final transformed matrix.

\section{Transmitting Phase:}

- Separate transformed image matrix into blocks of pixel values. Each block of pixel values contain 2 column of the transformed matrix.

- Initialize threads for each block of data and transmit the data.

\section{Decoding Phase:}

- Initialize a thread for each block of received data. Start threads for each block for reverse column transformation process.

- When a pair of elements in the same row is available transform them (reverse row transformation).

- If both column and row reverse transformation processes are completed then the thread disposed

\section{Performance ANALYsis USING ExPERIMENTAL RESULT}

We have operated the proposed progressive image transmission with concurrent computing on four different personal computers. The configurations of the computers were different and we tested number of color images of various dimensions. In Table IV, the time required for one stage of encoding and decoding for images for normal discrete wavelet transformation and concurrent computing are shown. Column A's represent the time required for concurrent wavelet transformation and column B's represent the time required for normal discrete wavelet transformation. All the values in the table are in millisecond. The images used here are lenna(512 x 512), moon(1986 x 1986), pepper(512 x 512), airplane(512 x 512), baboon(512 x 512), cornell_box $(300$ x 300).

The main objective of the proposed method is to reduce the browsing time of images with preserving the quality. The data presented in Table IV shows how much the encoding and decoding time has reduced by applying concurrent computing with comparison to normal sequential computing. Though the transmission time may vary because of transmission lines, the required time will be reduced much as the two main phases requires far less time. From our experimental result we found that the required time for encoding and decoding is up to $90 \%$ or more less than the sequential computing. The performance may vary on different machines but still it gives much better time overhead.

This method forms the output image with fractions. But as the fractions are parts of the original image without modification and it appears very fast the person can identify whether the image is the actual one or not within very short time. The required time to complete the whole process of image transmission for several images is shown in table II. Here all images here are color images. The table II shows that how much better performance is found after applying concurrent computing rather than sequential computing.

Generally, when the PSNR is $40 \mathrm{~dB}$ or larger, then the original image and the reconstructed image after transmission are virtually indistinguishable by human observers. In this case we found that with our method, it provides good level of details to the output. Comparing with other wavelet-based PIT schemes [2] the output is acceptable. We have tested several images and found good quality of the reconstructed image compared with other PIT schemes. C.C. Chang has shown the PSNR [2] of 'lenna' found with his proposed approach is 33.03. With our approach this measure has found 40.71. In Table III we have shown PSNR and MSE for different images.

TABLE II: COMPARISON OF REQUIRED TIME OF TRANSMISSION

\begin{tabular}{|c|c|c|}
\hline Images & $\begin{array}{c}\text { Transmission time with } \\
\text { concurrency(ms) }\end{array}$ & $\begin{array}{c}\text { Trans mission time } \\
\text { without } \\
\text { concurrency(ms) }\end{array}$ \\
\hline Lenna & 123.7 & 643.3 \\
\hline Baboon & 127.9 & 595.9 \\
\hline Cornell_box & 42.4 & 280 \\
\hline Pepper & 120.2 & 650.3 \\
\hline Airplane & 119.7 & 677.1 \\
\hline Moon & 1644.4 & 9524.3 \\
\hline
\end{tabular}


Table III: PSNR and MSE of reconstructed image

\begin{tabular}{|c|c|c|c|}
\hline Images & PSNR & MSE & PSNR[2] \\
\hline F14 & 41.44 & 4.66 & 42.56 \\
\hline Lenna & 40.71 & 5.52 & 33.03 \\
\hline Barbara & 36.34 & 16.88 & 37.16 \\
\hline Boat & 39.85 & 7.27 & 40.35 \\
\hline baboon & 38.97 & 9.61 & 38.46 \\
\hline
\end{tabular}

Table IV: Time comparison between normal discrete wavelet transformation and concurrent discrete wavelet transformation

\begin{tabular}{|c|c|c|c|c|c|c|c|c|c|c|c|c|c|c|c|c|}
\hline \multirow{3}{*}{$\begin{array}{c}\mathrm{CPU} \\
\text { Images }\end{array}$} & \multicolumn{4}{|c|}{$\begin{array}{c}\text { Intel Core } 2 \mathrm{CPU} \\
(2 \mathrm{GHz})\end{array}$} & \multicolumn{4}{|c|}{$\begin{array}{l}\text { AMD } 64 X 2 \text { Dual } \\
(2 \mathrm{GHz})\end{array}$} & \multicolumn{4}{|c|}{$\begin{array}{c}\text { Intel P4 } \\
(2.66 \mathrm{GHz})\end{array}$} & \multicolumn{4}{|c|}{ Intel Core 2 Duo (2.66 GHZ) } \\
\hline & \multicolumn{2}{|c|}{ Encode } & \multicolumn{2}{|c|}{ Decode } & \multicolumn{2}{|c|}{ Encode } & \multicolumn{2}{|c|}{ Decode } & \multicolumn{2}{|c|}{ Encode } & \multicolumn{2}{|c|}{ Decode } & \multicolumn{2}{|c|}{ Encode } & \multicolumn{2}{|c|}{ Decode } \\
\hline & A & B & A & B & A & B & A & B & A & B & A & B & A & B & A & B \\
\hline Lenna & 2.5 & 56.9 & 0.8 & 81.1 & 5.5 & 80 & 0.5 & 116 & 2.8 & 103 & 84.6 & 143.1 & 0.8 & 52.2 & 0.3 & 78.1 \\
\hline Pepper & 24.1 & 57.1 & 0.8 & 181.8 & 17.4 & 80.9 & 1.7 & 115.2 & 12.3 & 100.3 & 31.2 & 144.5 & 0.9 & 143.6 & 0.3 & 234.9 \\
\hline Airplane & 3.4 & 56.7 & 0.7 & 150.6 & 2.8 & 80.3 & 0.5 & 115.9 & 2.8 & 100.1 & 0.7 & 145.8 & 1.9 & 143.9 & 0.7 & 216.3 \\
\hline Baboon & 3.9 & 50.6 & 0.7 & 140.4 & 2.5 & 70.7 & 0.5 & 102.4 & 2.7 & 82.6 & 0.6 & 120.4 & 0.6 & 126.7 & 0.3 & 214.6 \\
\hline
\end{tabular}

In figure 5 shows time comparison graph between normal discrete wavelet transformation and concurrent discrete wavelet transformation of encoding phase for Intel Core2 Duo.

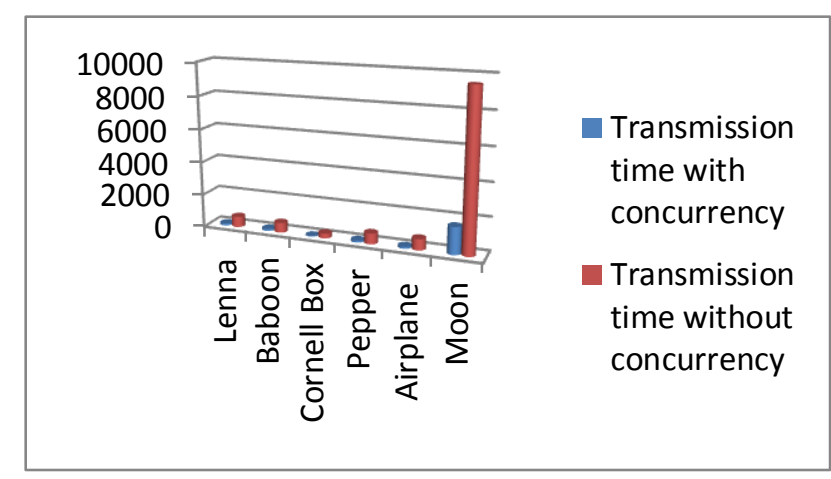

Figure 5. Time comparison between normal discrete wavelet transformation and concurrent discrete wavelet transformation graph of encoding phase.

\section{CONCLUSION}

Progressive image transmission (PIT) transmits the most significant portion of a picture, followed by its less important portions. The mechanism can be used in Web- based applications while users are browsing images. However, most PIT methods use the same pixel interpolation scheme for the entire picture, without considering the differences among image blocks. We have presented a method to enhance the progressive image transmission. The method uses concurrent computing on Discrete Wavelet Transformation to get the enhancement. This scheme is basically to reduce the image browsing time. Our method reconstructs the image with a few distortions and within far less time than the traditional one.

\section{REFERENCES}

[1] K H. Talukder and K. Harada, "Haar Wavelet Based Approach for Image Compression and Quality Assessment of Compressed Image", IAENG International Journal of Applied Mathematics, 36:1, IJAM_36_1_9.

[2] C C. Chang and T. C. Lu, "A Wavelet-based Progressive Digital Image Transmission Scheme", 
Proceedings of the First International Conference on Innovative Computing, Information and Control - Volume 2, 2006, pp. 681-684.

[3] K. H. Tzou, "Progressive Image Transmission: A Review and Comparison of Technique," Optical Engineering, Vol. 26, No. 7, 1987, pp. 581-589.

[4] R C. Chang, T. K. Shih, "Innovative Decomposition for Progressive 1Image Transmission ", Asian Journal of Health and Information Sciences, Vol. 1, No. 2, pp. 204-227, 2006.

[5] K H. Talukder and K. Harada, "A Scheme of Wavelet Based Compression of 2D Image", Proc. IMECS, Hong Kong, pp. 531-536, June 2006.

[6] J S. Walker, "A Primer on WAVELETS and Their Scientific Applications", Second Edition, University of Wisconsin Eau Claire, Wisconsin, U.S.A.

[7] G K. Wallace, "The JPEG Still Picture Compression Standard, Comm. “, ACM, vol. 34, no. 4, April 1991, pp. 30-44.

[8] A. Wesley, B. Ari, Mordechai, "Principles of Concurrent and Distributed Programming (2nd ed.)", By ISBN 978-0-321-31283-9.

[9] G Strang and T. Nguyen, "Wavelets and Filter Banks", Wellesley-Cambridge Press, Wellesley, MA,

1996,http://www.math.mit.edu/ gs/books/wfb.html.

[10] S. Saha, "Image Compression - from DCT to Wavelets : A Review", The ACM Student Magazine,

http://www.acm.org/crossroads/xrds63/sahaimgcodi ng.html

\section{AUTHORS PROFILE}

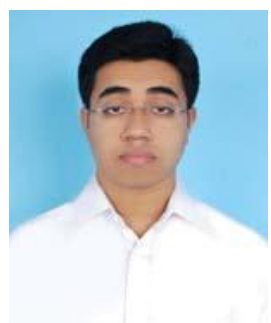

Md. Rifat Ahmmad Rahsid has completed his B.Sc Engg in Computer Science and Engineering (CSE) from Khulna University (KU) Khulna, Bangladesh. His research interest is mainly focused on Image processing, Data mining and Knowledge discovery, AI, Software Engineering, HCI, Ubiquitous Computing and Cognitive and Computational Neuroscience. Currently he is a Lecturer in the Dept. of Computer Science and Engineering (CSE), Bangladesh University of Business and Technology (BUBT).

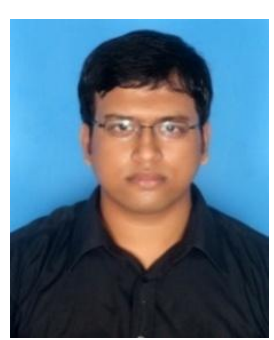

Mir Tafseer Nayeem obtained his BSc degree in Computer Science and Information Technology from Islamic University of Technology (IUT), Gazipur, Bangladesh in 2011. He received the OIC (Organization of the Islamic Conference) scholarship for three years during his BSc studies. His research interest is mainly focused on AI, Ad Hoc Networks, Software Engineering, HCI, Image processing, Web Mining, Ubiquitous Computing and Cognitive and Computational Neuroscience. Currently he is a Lecturer in the Dept. of Computer Science and Engineering (CSE), Bangladesh University of Business and Technology (BUBT).

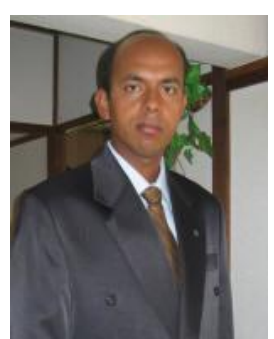

Kamrul Hasan Talukder has completed his B.Sc Engg in Computer Science and Engineering (CSE) from Khulna University (KU) Khulna, Bangladesh in 1999. $\mathrm{He}$ also completed his $\mathrm{MSc}$ (Computer Science, by research) from National University of Singapore (NUS), Singapore, in 2004. He is an Associate Professor in the Department of Computer Science and Engineering (CSE) Khulna 
University (KU), Khulna Bangladesh. Currently he is doing post doctorial in the Dept. of Information Engineering, Hiroshima University, Japan. His research interest is mainly focused on Image Processing, Formal Verification and Software Engineering. He has published more than 50 research publications in various National, International conferences, proceedings and Journals.

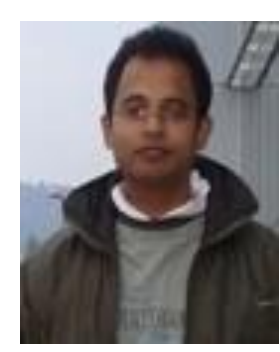

\section{Md. Saddam Hossain Mukta} obtained his M.sc degree in Computer science from University of Trento, Italy where he was receiving Opera Universita Scholarship and earned a B.Sc degree in Computer Science and Information Technology from Islamic University of Technology (IUT), Gazipur, Bangladesh in 2006. His research interest is mainly focused on Semantic web, Social computing, Software Engineering, HCI, Image processing, Web Mining and Data \& knowledge management. Currently he is a Lecturer in the Dept. of Computer Science and Engineering (CSE), Bangladesh University of Business and Technology (BUBT). 